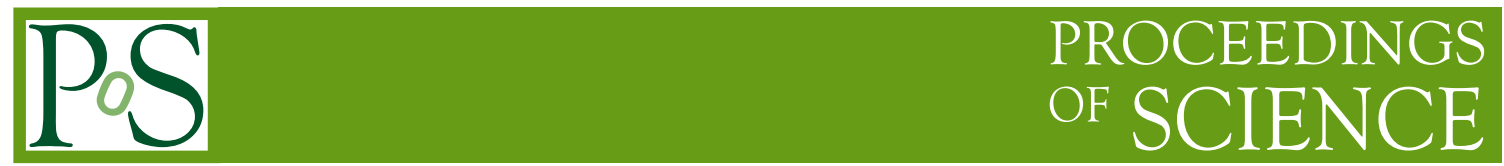

\title{
VBS/VBF measurements (without photons) at ATLAS
}

\author{
Karolos Potamianos ${ }^{a, \dagger, *}$ \\ ${ }^{a}$ Department of Physics, University of Oxford, $U K$ \\ E-mail: karolos.potamianos@cern.ch
}

Electroweak symmetry breaking explains the origin of the masses of elementary particles via their interactions with the Higgs field. Besides the measurements of the Higgs boson properties, the study of the scattering of vector bosons at the Large Hadron Collider (LHC) allows to probe the nature of electroweak symmetry breaking with an unprecedented sensitivity. We report on the latest Vector Boson Scattering (VBS) and Vector Boson Fusion (VBF) measurements from the ATLAS Collaboration at the LHC using events with no photons in the final state. This review focuses on the most recent ATLAS VBS/VBF measurements using the full dataset $\left(139 \mathrm{fb}^{-1}\right)$ collected by the ATLAS detector during LHC Run-2 (2015-2018).

The Ninth Annual Conference on Large Hadron Collider Physics - LHCP2021

7-12 June 2021

Online

\footnotetext{
${ }^{\dagger}$ On behalf of the ATLAS Collaboration

* Speaker
} 


\begin{tabular}{l|c|c|c} 
Process & $\begin{array}{c}\text { Luminosity } \\
{\left[\mathrm{fb}^{-1}\right]}\end{array}$ & Significance & Ref. \\
\hline$p p \rightarrow Z j j$ & 139 & & {$[2]$} \\
$p p \rightarrow Z Z j j$ & 139 & $5.5 \sigma$ & {$[3]$} \\
$\gamma \gamma \rightarrow W^{ \pm} W^{\mp}$ & 139 & $8.4 \sigma$ & {$[4]$} \\
$p p \rightarrow W^{ \pm} W^{ \pm} j j$ & 36 & $6.5 \sigma$ & {$[5]$} \\
$p p \rightarrow W^{ \pm} Z j j$ & 36 & $5.3 \sigma$ & {$[6]$} \\
$p p \rightarrow V V j j$ semileptonic & 36 & $2.7 \sigma$ & {$[7]$}
\end{tabular}

Table 1: Summary of ATLAS Run-2 VBF/VBS measurements with no photons in the final state.

\section{Introduction and experimental context}

Electroweak symmetry breaking (EWSB) plays a central role in the Standard Model (SM) of particle physics, as it explains the origin of elementary particle masses via the interactions of each particle with the Higgs field.

Measurements that exploit the weak vector boson scattering (VBS) and weak vector boson fusion (VBF) processes have become increasingly prevalent at the Large Hadron Collider (LHC) in the last few years. These measurements rely on theoretical predictions to accurately model the electroweak processes that are sensitive to the VBF and VBS production mechanisms.

Monte Carlo (MC) event generators are used to optimise the event selection and to extract the electroweak signal from the dominant background, with the signal extraction typically performed using fits to kinematic spectra. However, the theoretical predictions from different event generators do not agree, both in the overall production rate as well as in the kinematic properties of the final state. Model-independent measurements that directly probe the kinematic properties of VBS and VBF are therefore crucial, to determine which event generators can be used reliably in physics analysis at the LHC experiments

Furthermore, VBS at high energies is subject to delicate cancellations between terms [1], making these processes very sensitive to shifts in the trilinear or quartic gauge couplings of the vector bosons. Therefore, $p p \rightarrow V(V) j j$ is a fundamental probe of the $S U(2)_{L} \times U(1)_{Y}$ symmetry of the Standard Model (SM) of particle physics.

This review focuses on the most recent ATLAS VBS/VBF measurements with no photons in the final state using the full dataset $\left(139 \mathrm{fb}^{-1}\right)$ collected by the ATLAS detector during LHC Run-2 (2015-2018). Table 1 shows an overview of the ATLAS Run-2 VBF/VBS measurements with no photons in the final state.

\section{Description of the experimental analyses}

ATLAS differentially measures the electroweak and inclusive production cross-section of two jets in association with a $Z$ boson, $Z j j$ [2] in the $Z \rightarrow \ell^{+} \ell^{-}$decay channel $(\ell=e, \mu)$ as a function of four observables: the dijet invariant mass (shown in Fig. 1), the rapidity interval spanned by the two jets, the signed azimuthal angle between the two jets, and the transverse momentum of the dilepton pair. The data, corrected for the effects of detector inefficiency and resolution, are sufficiently precise to distinguish between different state-of-the-art theoretical predictions calculated using Powheg+Pythia8, Herwig7+VbFnlo and Sherpa 2.2. The differential cross-sections are used to search for anomalous weak-boson self-interactions using a dimension-six (dim-6) effective 


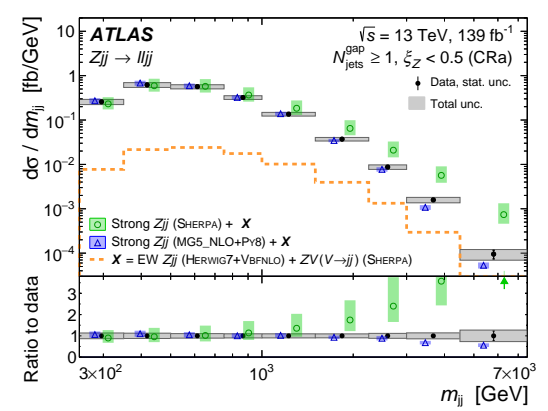

(a) Inclusive $Z_{j j}$

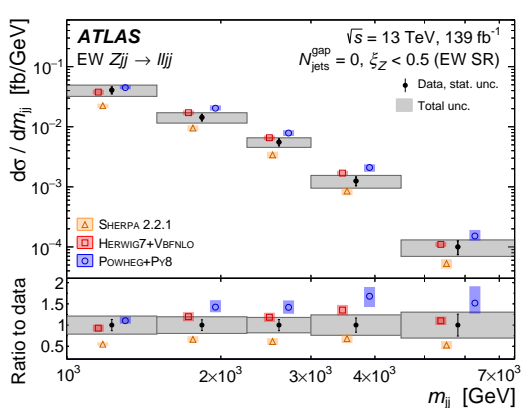

(b) $\mathrm{EW} Z j j$

Fig. 1: Differential cross-sections for (a) inclusive and (b) EW $Z_{j j} j$ production as a function of $m_{j j}$. The unfolded data are shown as black points, with the statistical uncertainty represented by an error bar and the total uncertainty represented as a grey band. The data are compared with theoretical predictions constructed from different strong $Z j j$ predictions provided by SHERPA (green) and MG5_NLO+Py8 (blue). Uncertainty bands are shown for the two theoretical predictions. Each theory prediction is slightly offset from the bin center to avoid overlap. Source: [2].

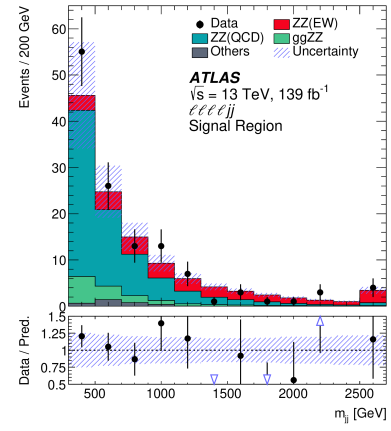

(a) $m_{j j}$

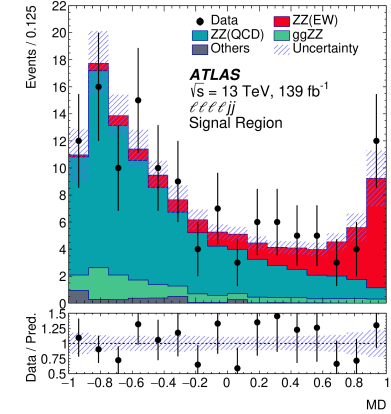

(b) Multivariate discriminant

Fig. 2: Observed and expected (a) $m_{j j}$ and (b) multivariate discriminant distributions in the $\ell \ell \ell \ell j j$ signal region. The error bands include the expected experimental and theoretical uncertainties. The contributions from the QCD and EW production of $Z Z j j$ events are scaled by the observed normalisation factors in the statistical fit to the combined channel. All the minor backgrounds are summed together as 'Others'. The last bin includes the overflow events. The open arrows represent the out-of-range markers. Source: [3].

field theory. The measurement of the signed azimuthal angle between the two jets is found to be particularly sensitive to the interference between the SM and dim- 6 scattering amplitudes and provides a direct test of charge-conjugation and parity invariance in the weak-boson self-interactions.

Among all processes related to vector-boson scattering, the electroweak production of two jets and a $Z$-boson pair via VBS, $Z Z j j$, is a rare and important one. ATLAS reports the first observation of this process [3]. Two different final states originating from the decays of the Z-boson pair, one containing four charged leptons and the other containing two charged leptons and two neutrinos, are considered. Fig. 2 shows the observed and expected distributions for $m_{j j}$ and the multivariate discriminant trained to isolate $Z Z(\mathrm{EW}) j j$ events in the $\ell \ell \ell \ell j j$ signal region. The hypothesis of no 


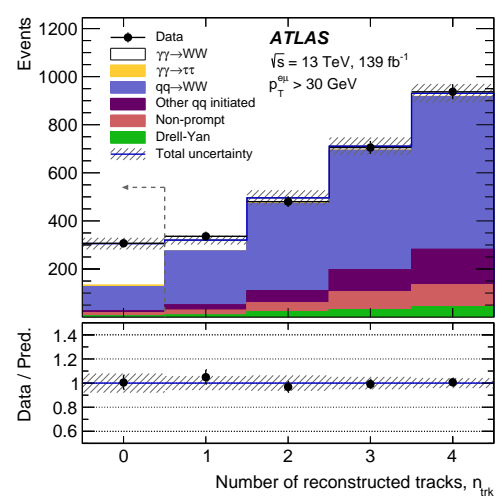

Fig. 3: The distribution of the number of tracks associated with the interaction vertex is shown. The fitted normalisation factors and nuisance parameters have been used. The $\gamma \gamma \rightarrow W W$ signal region requires a selection of $n_{\text {track }}=0$, as indicated by the vertical dashed line. The total uncertainties are shown as hatched bands. The lower panel shows the ratio of the data to the prediction, with the total uncertainty displayed as a hatched band. Source: [4].

electroweak production is rejected with a statistical significance of $5.5 \sigma$ (4.3 $\sigma$ expected), and the measured cross-section for electroweak production is consistent with the SM prediction. The EW $Z Z j j$ cross-section in the combined fiducial volume is found to be $0.82 \pm 0.21 \mathrm{fb}$ and is one of the smallest cross-sections measured by ATLAS. The cross-sections for inclusive production of $Z Z j j$ are also measured in the two final states.

$$
\begin{aligned}
& \sigma\left(Z Z_{j j} \mid \ell \ell \ell \ell j j\right)=1.27 \pm 0.12(\text { stat }) \pm 0.02(\text { theo }) \pm 0.07(\exp ) \pm 0.01(\mathrm{bkg}) \pm 0.03(\text { lumi }) \\
& \sigma(Z Z j j \mid \ell \ell v v j j)=1.22 \pm 0.30(\text { stat }) \pm 0.04(\text { theo }) \pm 0.06(\exp ) \pm 0.16(\mathrm{bkg}) \pm 0.03 \text { (lumi) }
\end{aligned}
$$

ATLAS observed photon-induced production of $W$-boson pairs, $\gamma \gamma \rightarrow W W$. The measurement is performed selecting one electron and one muon, corresponding to the decay $W W \rightarrow e^{ \pm} v \mu^{\mp} v$. The background-only hypothesis is rejected with a significance of $8.4 \sigma$. A cross section for the $\gamma \gamma \rightarrow W W$ process of $3.13 \pm 0.31$ (stat.) \pm 0.28 (syst.) fb is measured in a fiducial volume close to the acceptance of the detector, requiring an electron and a muon of opposite signs with large dilepton transverse momentum and no additional charged particles (see Fig. 3).

\section{Conclusions}

In summary, several recent measurements of VBF and VBS processes performed by ATLAS using the full Run-2 dataset have been presented. These measurements are in line with the SM expectations and help further test the predictions from various Monte Carlo generators. Some of the processes have been measured differentially, while some others not yet. In addition to providing more precise differential measurements, LHC Run-3 (and later runs at the High Luminosity LHC, HL-LHC) will enable focusing on searches for anomalous couplings (TGC and QGC) using EFT approaches. Furthermore, they will enable probing the polarisation state of the vector bosons, and extracting the fraction of events with longitudinally-polarised vector bosons, which is very sensitive to new physics and is probing the core of EWSB. This motivates efforts to devise improved analysis techniques during the Run 3 and the HL-LHC. 


\section{References}

[1] M. Szleper, "The Higgs boson and the physics of $W W$ scattering before and after Higgs discovery," [arXiv: 1412.836 [hep-ph]].

[2] G. Aad et al. [ATLAS Collaboration], "Differential cross-section measurements for the electroweak production of dijets in association with a $Z$ boson in proton-proton collisions at ATLAS,” Eur. Phys. J. C 81 (2021) no.2, 163 doi:10.1140/epjc/s10052-020-08734-w [arXiv: 2006.15458 [hep-ex]].

[3] G. Aad et al. [ATLAS Collaboration], "Observation of electroweak production of two jets and a Z-boson pair with the ATLAS detector at the LHC," [arXiv: 2004.10612 [hep-ex]].

[4] G. Aad et al. [ATLAS Collaboration], "Observation of photon-induced $W^{+} W^{-}$production in $p p$ collisions at $\sqrt{s}=13 \mathrm{TeV}$ using the ATLAS detector," Phys. Lett. B 816 (2021), 136190 doi:10.1016/j.physletb.2021.136190 [arXiv: 2010.04019 [hep-ex]].

[5] M. Aaboud et al. [ATLAS Collaboration], "Observation of electroweak production of a samesign $W$ boson pair in association with two jets in $p p$ collisions at $\sqrt{s}=13 \mathrm{TeV}$ with the ATLAS detector," Phys. Rev. Lett. 123 (2019) no.16, 161801 doi:10.1103/PhysRevLett.123.161801 [arXiv: 1906.03203 [hep-ex]].

[6] M. Aaboud et al. [ATLAS Collaboration], "Observation of electroweak $W^{ \pm} Z$ boson pair production in association with two jets in $p p$ collisions at $\sqrt{s}=13 \mathrm{TeV}$ with the ATLAS detector," Phys. Lett. B 793 (2019), 469-492 doi:10.1016/j.physletb.2019.05.012 [arXiv: 1812.09740 [hep-ex]].

[7] G. Aad et al. [ATLAS Collaboration], "Search for the electroweak diboson production in association with a high-mass dijet system in semileptonic final states in $p p$ collisions at $\sqrt{s}=13 \mathrm{TeV}$ with the ATLAS detector," Phys. Rev. D 100 (2019) no.3, 032007 doi:10.1103/PhysRevD.100.032007 [arXiv: 1905.07714 [hep-ex]]. 